\title{
Minimum rank with zero diagonal
}

\section{Authors}

Cheryl Grood, Johannes Harmse, Leslie Hogben, Thomas J. Hunter, Bonnie Jacob, Andrew Klimas, and Sharon McCathern 


\title{
MINIMUM RANK WITH ZERO DIAGONAL*
}

\author{
CHERYL GROOD ${ }^{\dagger}$, JOHANNES HARMSE ${ }^{\ddagger}$, LESLIE HOGBEN ${ }^{\S}$, THOMAS J. HUNTER ${ }^{\dagger}$, \\ BONNIE JACOB $\llbracket$, ANDREW KLIMAS", AND SHARON MCCATHERN ${ }^{\ddagger}$
}

\begin{abstract}
Associated with a simple graph $G$ is a family of real, symmetric zero diagonal matrices with the same nonzero pattern as the adjacency matrix of $G$. The minimum of the ranks of the matrices in this family is denoted $\operatorname{mr}_{0}(G)$. We characterize all connected graphs $G$ with extreme minimum zero-diagonal rank: a connected graph $G$ has $\operatorname{mr}_{0}(G) \leq 3$ if and only if it is a complete multipartite graph, and $\mathrm{mr}_{0}(G)=|G|$ if and only if it has a unique spanning generalized cycle (also called a perfect $[1,2]$-factor). We present an algorithm for determining whether a graph has a unique spanning generalized cycle. In addition, we determine maximum zero-diagonal rank and show that for some graphs, not all ranks between minimum and maximum zero-diagonal ranks are allowed.
\end{abstract}

Key words. Zero-Diagonal, Minimum rank, Maximum nullity, Zero forcing number, Perfect $[1,2]$-factor, Spanning generalized cycle, Matrix, Graph.

AMS subject classifications. 05C50, 05C70, 15A03, 15A18, $15 \mathrm{~B} 57$.

1. Introduction. Minimum rank problems focus on the minimum rank of a set of matrices that are described by a particular graph. The classic minimum rank problem examines real symmetric matrices whose diagonal is allowed to be free, and it has been studied extensively, along with its generalizations to other fields. Surveys of known results and the motivation for the minimum rank problem appear in $\underline{3}$ and 4. Further generalizations of the problem have been considered, including to skew-symmetric matrices $[8$ and to graphs that allow loops [2], 7], [10].

In this paper, we consider the minimum rank of a set of real symmetric matrices described by a simple graph, as in the classic case, but we restrict the diagonal entries

\footnotetext{
${ }^{*}$ Received by the editors on September 8, 2013. Accepted for publication on June 2, 2014. Handling Editor: Bryan L. Shader.

$\dagger$ Department of Mathematics and Statistics, Swarthmore College, Swarthmore, PA 19081, USA (cgrood1@swarthmore.edu, thunter1@swarthmore.edu).

${ }_{\ddagger}$ Department of Mathematics and Physics, Azusa Pacific University, Azusa, CA 92037, USA (jharmse@apu.edu, smccathern@apu.edu).

$\S$ Department of Mathematics, Iowa State University, Ames, IA 50011, USA (lhogben@iastate.edu), and American Institute of Mathematics, 360 Portage Ave, Palo Alto, CA 94306 (hogben@aimath.org).

IScience and Mathematics Department, National Technical Institute for the Deaf at Rochester Institute of Technology, Rochester, NY 14623, USA (bcjntm@rit.edu).

"Department of Mathematics, Xavier University of Louisiana, New Orleans, LA 70125, USA (aklimas@xula.edu).
} 
to be zero. Such matrices generalize the adjacency matrix, and can be considered as adjacency matrices of weighted simple graphs (where the edge weight reflects the value of the entry). This problem has connections to the problem of skew minimum rank and is a special case of minimum rank for graphs that allow loops; there are also connections to the study of perfect [1,2]-factors of graphs, also known as spanning generalized cycles. In the study of standard minimum rank, graphs with very small or large minimum rank have been characterized. We prove analogous results for minimum zero-diagonal rank: In Section 2, we determine all graphs with minimum zero-diagonal rank at most 3. In Section 3, we characterize graphs whose associated matrices are all nonsingular, and give an algorithm for testing whether a graph has this property by determining whether it has a unique spanning generalized cycle (i.e., a unique perfect [1,2]-factor). In Section 4, we investigate which matrix ranks are allowed by a given graph $G$ and determine the maximum zero-diagonal rank. Section 5 contains concluding remarks.

1.1. Notation and terminology. A graph $G$ is a pair $(V(G), E(G))$ of sets where the set of vertices $V(G)$ is finite and nonempty, and each element of the set of edges $E(G)$ consists of a set of two distinct elements of $V(G)$. The order of a graph $G$, denoted $|G|$, is the number of its vertices, i.e., $|V(G)|$. Throughout we denote by $P_{n}, C_{n}$, and $K_{n}$ the path, cycle, and complete graph on $n$ vertices, respectively; $K_{n_{1}, n_{2}, \ldots, n_{r}}$ (for $r \geq 2$ and $n_{i} \geq 1$ ) designates the complete multipartite graph containing $n_{i}$ vertices in the $i$ th partite set, $i=1, \ldots, r$. A path $P_{n}$ or cycle $C_{n}$ is called odd or even according as $n$ is odd or even. A Hamilton cycle of a graph is a cycle that contains every vertex of the graph, and we call a graph Hamiltonian if it contains a Hamilton cycle. A chord of a cycle is an edge whose endpoints are nonadjacent vertices of the cycle. A graph $G$ is connected if there is a path between any two distinct vertices; a connected component $G^{\prime}$ of $G$ is a connected subgraph of $G$ that is not properly contained in any connected subgraph of $G$.

The following methods of obtaining a new graph from given graphs are used in this paper. The union of $G_{i}=\left(V_{i}, E_{i}\right), i=1, \ldots, h$, is $\bigcup_{i=1}^{h} G_{i}=\left(\cup_{i=1}^{h} V_{i}, \cup_{i=1}^{h} E_{i}\right)$; if the $V_{i}$ are pairwise disjoint, then the union is denoted $\bigcup_{i=1}^{\cdot h} G_{i}$. If $\{u, v\}$ is an edge in a graph $G$, the subdivision of $\{u, v\}$ yields a graph with one new vertex $w$, and with an edge set obtained by replacing $\{u, v\}$ by two new edges $\{u, w\}$ and $\{w, v\}$. A graph $H$ is a subgraph of a graph $G$ if $V(H) \subseteq V(G)$ and $E(H) \subseteq E(G)$. The subgraph of $G$ induced by $U \subset V(G)$ is the subgraph of $G$ with vertex set $U$ and with edge set given by $\{\{i, j\} \in E(G) \mid i, j \in U\}$; it is denoted $G[U]$. If $v \in V(G)$, we write $G-v$ for the subgraph of $G$ induced by $V(G) \backslash\{v\}$.

Following the literature, for a symmetric $n \times n$ matrix $A$ we define the graph of 
the matrix $A$, denoted $\mathcal{G}(A)$, to be the graph with vertices $\{1,2, \ldots, n\}$ and edges $\left\{\{i, j\} \mid a_{i j} \neq 0,1 \leq i<j \leq n\right\}$; note that the diagonal entries of $A$ are irrelevant in determining its associated graph $\mathcal{G}(A)$. One can easily observe that many different $n \times n$ symmetric matrices yield the same graph. We denote the set of symmetric matrices whose graph is $G$ by $\mathcal{S}(G)$; that is, $\mathcal{S}(G)=\left\{A \in \mathbb{R}^{n \times n} \mid A^{T}=A, \mathcal{G}(A)=G\right\}$. The standard minimum rank problem uses the set $\mathcal{S}(G)$; it has been studied at length. The minimum rank of a graph $G$ is defined as $\operatorname{mr}(G)=\min \{\operatorname{rank} A \mid A \in \mathcal{S}(G)\}$, and the maximum nullity of $G$ is given by $\mathrm{M}(G)=\max \{$ null $A \mid A \in \mathcal{S}(G)\}$.

In this paper, we restrict ourselves to the subset of $\mathcal{S}(G)$ consisting of zero diagonal matrices:

$$
\mathcal{S}_{0}(G)=\left\{A \in \mathcal{S}(G) \mid a_{i i}=0,1 \leq i \leq n\right\}
$$

We are concerned with finding the minimum rank over this set of matrices. The minimum zero-diagonal rank of a graph $G$ is

$$
\operatorname{mr}_{0}(G)=\min \left\{\operatorname{rank} A \mid A \in \mathcal{S}_{0}(G)\right\}
$$

and the maximum zero-diagonal nullity of $G$ is

$$
\mathrm{M}_{0}(G)=\max \left\{\operatorname{null} A \mid A \in \mathcal{S}_{0}(G)\right\} .
$$

Since $\mathcal{S}_{0}(G) \subseteq \mathcal{S}(G)$, it is clear that $\operatorname{mr}(G) \leq \operatorname{mr}_{0}(G)$. Just as in the standard minimum rank problem, for $A \in \mathcal{S}_{0}(G)$ the principal submatrix $A[U]$ (the part of $A$ contained in rows and columns indexed by $U$ ) is associated with the induced subgraph $G[U]$. The statements in the next observation are analogous to those in the standard minimum rank case and are justified by the same reasoning.

\section{OBSERVATION 1.1}

1. If $H$ is an induced subgraph of $G$, then $\operatorname{mr}_{0}(H) \leq \operatorname{mr}_{0}(G)$.

2. A graph $G$ has no edges if and only if $\operatorname{mr}_{0}(G)=0$.

3. If the connected components of $G$ are $G_{1}, G_{2}, \ldots, G_{t}$, then

$$
\mathrm{mr}_{0}(G)=\sum_{i=1}^{t} \operatorname{mr}_{0}\left(G_{i}\right) \text {. }
$$

1.2. Generalized cycles. A cycle $\left(v_{1}, v_{2}, \ldots, v_{k}\right)$ in a graph $G$ is a subgraph with distinct vertices $v_{1}, v_{2}, \ldots, v_{k}, k \geq 3$, and edges $\left\{v_{1}, v_{2}\right\},\left\{v_{2}, v_{3}\right\}, \ldots,\left\{v_{k-1}, v_{k}\right\}$, $\left\{v_{k}, v_{1}\right\}$; a cycle with $k$ vertices is called a $k$-cycle. A generalized cycle of $G$ is a subgraph of $G$ whose connected components are either single edges (meaning an edge and its two endpoints) or cycles. The order of a generalized cycle is the number of vertices in the generalized cycle; a generalized cycle of order $|G|$ is also called a 
spanning generalized cycle. Spanning generalized cycles appear in the literature under a variety of other names, including perfect $[1,2]$-factors [6] and linear subgraphs [5].

Given a generalized cycle $\mathcal{C}, \operatorname{nc}(\mathcal{C})$ is the number of distinct cycles in $\mathcal{C}$, and ne $(\mathcal{C})$ is the number of even components of $\mathcal{C}$, i.e., the number of cycles of even order at least 4 plus the number of edges. The set of all generalized cycles of order $k$ of a graph $G$ is denoted $\operatorname{cyc}_{k}(G)$. With a generalized cycle $\mathcal{C}$, we can associate a permutation $\pi_{\mathcal{C}}$ of the vertices of $\mathcal{C}$ as follows: For each cycle in $\mathcal{C}$, fix an orientation and then associate a directed graph cycle $\left(v_{j_{1}}, v_{j_{2}}, \ldots, v_{j_{\ell}}\right)$ with the cyclic permutation $\left(v_{j_{1}} v_{j_{2}} \cdots v_{j_{\ell}}\right)$. Each edge component $\left\{v_{i_{1}}, v_{i_{2}}\right\}$ of $\mathcal{C}$ is associated with the 2-cycle $\left(v_{i_{1}} v_{i_{2}}\right)$. The permutation $\pi_{\mathcal{C}}$ is then defined to be the product of these associated permutation cycles. Note that there are $2^{\text {nc }(\mathcal{C})}$ different choices for the orientation of the cycles of $\mathcal{C}$, and each choice yields a permutation that has the same sign as $\pi_{\mathcal{C}}$, namely $(-1)^{\text {ne }(\mathcal{C})}$. We denote the sum of all $k \times k$ principal minors of an $n \times n$ matrix $A=\left[a_{i j}\right] \in \mathcal{S}_{0}(G)$ by $S_{k}(A)$, and [5. Theorem 3] shows that $S_{k}(A)$ can be computed using generalized cycles:

$$
S_{k}(A)=\sum_{\mathcal{C} \in \operatorname{cyc}_{k}(\mathcal{G}(A))}(-1)^{\operatorname{ne}(\mathcal{C})} 2^{\operatorname{nc}(\mathcal{C})} a_{i_{1} \pi_{\mathcal{C}}\left(i_{1}\right)} \ldots a_{i_{k} \pi_{\mathcal{C}}\left(i_{k}\right)}
$$

where the sum over the empty set is zero. In particular, (1.1) allows us to express $S_{n}(A)=\operatorname{det} A$ using the spanning generalized cycles. The fact that $G$ has no loops immediately implies $S_{1}(A)=0$, which is also easily verifiable by noting that all the diagonal entries of $A \in \mathcal{S}_{0}(G)$ are zero.

EXAMPLE 1.2. We give an example of using (1.1) to compute the characteristic polynomial $A, p_{A}(x)=x^{n}-S_{1}(A) x^{n-1}+S_{2}(A) x^{n-2}+\cdots+(-1)^{n-1} S_{n-1}(A) x+$ $(-1)^{n} S_{n}(A)$, for an arbitrary $A \in \mathcal{S}_{0}(G)$. Let $G$ be the paw graph shown in Figure 1.1. Then $A \in \mathcal{S}_{0}(G)$ has the form $A=\left[\begin{array}{cccc}0 & a_{12} & 0 & 0 \\ a_{12} & 0 & a_{23} & a_{24} \\ 0 & a_{23} & 0 & a_{34} \\ 0 & a_{24} & a_{34} & 0\end{array}\right]$. We can use (1.1) to compute $S_{k}(A)$ for $k=2,3,4$. For $k=2$, the generalized cycles of order 2 are the edges, $\{1,2\},\{2,3\},\{2,4\},\{3,4\}$, and $S_{2}(A)=-a_{12}^{2}-a_{23}^{2}-a_{24}^{2}-a_{34}^{2}$. For $k=3$, the only generalized cycle of order 3 is the 3 -cycle $(2,3,4)$, and $S_{3}(A)=2 a_{23} a_{34} a_{24}$. For $k=4$, the only generalized cycle of order 4 is the union of two disjoint edges $\{1,2\}$ and $\{3,4\}$, and $S_{4}(A)=a_{12}^{2} a_{34}^{2}$. Thus, $p_{A}(x)=x^{4}-\left(a_{12}^{2}+a_{23}^{2}+a_{24}^{2}+a_{34}^{2}\right) x^{2}-$ $2 a_{23} a_{34} a_{24} x+a_{12}^{2} a_{34}^{2}$.

REMARK 1.3. If $G$ has a unique spanning generalized cycle, then by (1.1), $\operatorname{det} A=$ $S_{n}(A) \neq 0$ for all $A \in \mathcal{S}_{0}(G)$, so $\operatorname{mr}_{0}(G)=|G|$.

We explain the next result, which is well known, because of its importance to 


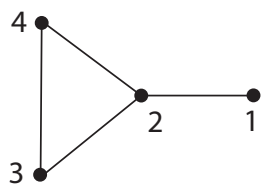

FIG. 1.1. The paw graph.

many of the proofs that follow.

REMARK 1.4. Let $G$ be a graph of order $n$ and $1 \leq m \leq n$. If $G$ has no generalized cycle of order $k$ for all $k>m$, then $\operatorname{mr}_{0}(G) \leq m$.

To see this, observe that if $G$ has no generalized cycle of order $k$, then for each $A \in \mathcal{S}_{0}(G)$, the coefficient of $x^{n-k}$ in $p_{A}(x)$ is zero. So if $G$ has no generalized cycle of order $k$ for all $k>m$, then for all $A \in \mathcal{S}_{0}(G)$

$$
\begin{aligned}
p_{A}(x) & =x^{n}-S_{1}(A) x^{n-1}+\cdots+(-1)^{m} S_{m}(A) x^{n-m} \\
& =\left(x^{m}-S_{1}(A) x^{m-1}+\cdots+(-1)^{m} S_{m}(A)\right) x^{n-m} .
\end{aligned}
$$

Since the algebraic and geometric multiplicities of an eigenvalue of a real symmetric matrix are equal, null $A \geq n-m$, and consequently, rank $A \leq m$. Thus, $\operatorname{mr}_{0}(G) \leq m$.

A matching in a graph $G$ is a set of edges $\left\{v_{1}, u_{1}\right\}, \ldots,\left\{v_{k}, u_{k}\right\}$ such that all the vertices are distinct. Note that a matching with $k$ edges is associated with a generalized cycle of order $2 k$. A perfect matching in a graph $G$ is a matching that includes all vertices of $G$. A maximum matching in $G$ is a matching with the maximum number of edges among all matchings in $G$. The matching number, denoted match $(G)$, is the number of edges in a maximum matching.

Observation 1.5. A graph $G$ has a generalized cycle of order $2 \operatorname{match}(G)$.

Proposition 1.6

$$
\operatorname{mr}_{0}\left(P_{n}\right)= \begin{cases}n & \text { if } n \text { is even; } \\ n-1 & \text { if } n \text { is odd. }\end{cases}
$$

Proof. If $n$ is even, then $P_{n}$ has a unique spanning generalized cycle associated with its unique perfect matching (consisting of every other edge starting at one end), so $\operatorname{mr}_{0}\left(P_{n}\right)=n$. If $n$ is odd then $n-1=\operatorname{mr}_{0}\left(P_{n-1}\right) \leq \operatorname{mr}_{0}\left(P_{n}\right)$. Since $P_{n}$ has no spanning generalized cycle, by $\operatorname{Remark} 1.4 \operatorname{mr}_{0}\left(P_{n}\right) \leq n-1$.

\section{Proposition 1.7.}

$$
\operatorname{mr}_{0}\left(C_{n}\right)= \begin{cases}n & \text { if } n \text { is odd } \\ n-2 & \text { if } n \text { is even } .\end{cases}
$$


Proof. If $n$ is odd then $C_{n}$ has a unique spanning generalized cycle, namely $C_{n}$ itself, so $\operatorname{mr}_{0}\left(C_{n}\right)=n$. Let $n$ be even (so $n \geq 4$ ). Observe that $P_{n-1}$ is an induced subgraph of $C_{n}$ and $n-1$ is odd, so $n-2=\operatorname{mr}_{0}\left(P_{n-1}\right) \leq \operatorname{mr}_{0}\left(C_{n}\right)$. If $n \equiv 0 \bmod 4$, then the adjacency matrix of $C_{n}$ has rank $n-2$; if $n \equiv 2 \bmod 4$, then the adjacency matrix of $C_{n}$ with one symmetric pair of 1 s replaced by -1 s has rank $n-2$. $\square$

1.3. Applying known results to compute minimum zero-diagonal rank. In this section, we survey results from earlier work on related problems and apply these results to compute minimum zero-diagonal rank.

Remark 1.8. Since $\operatorname{mr}_{0}(G) \geq \operatorname{mr}(G)$, the existence of a matrix $A \in \mathcal{S}_{0}(G)$ such that $\operatorname{rank} A=\operatorname{mr}(G)$ implies $\operatorname{mr}_{0}(G)=\operatorname{mr}(G)$. For example, the matrix constructed to realize minimum rank for the sth hypercube $Q_{s}$ in [1, Theorem 3.1] has zero diagonal for $s \geq 2$. Thus, $\operatorname{mr}_{0}\left(Q_{s}\right)=\operatorname{mr}\left(Q_{s}\right)=2^{s-1}$ for $s \geq 2$. Equality is not achieved for $s=1$, because $Q_{1}=P_{2}$.

A loop graph is a graph that allows loops but not multiple edges. More formally, a loop graph $\hat{G}=(\hat{V}, \hat{E})$ is a set of vertices $\hat{V}$ together with an edge set $\hat{E}$ of two-element multisets of vertices (note that a loop graph need not actually have any loops). For a loop graph $\hat{G}=(\hat{V}, \hat{E}), \mathcal{S}_{\ell}(\hat{G})=\left\{A \in \mathbb{R}^{n \times n} \mid A^{T}=A\right.$ and $\left.a_{i j} \neq 0 \Leftrightarrow\{i, j\} \in \hat{E}\right\}$ and $\operatorname{mr}_{\ell}(\hat{G})=\min \left\{\operatorname{rank} A \mid A \in \mathcal{S}_{\ell}(\hat{G})\right\}$. Observe that if a graph $G=(V(G), E(G))$ is viewed as a loop graph $\hat{G}$ with no loops, i.e., $\hat{V}=V(G)$ and $\hat{E}=E(G)$, then $\mathcal{S}_{\ell}(\hat{G})=\mathcal{S}_{0}(G)$, and thus, $\operatorname{mr}_{\ell}(\hat{G})=\operatorname{mr}_{0}(G)$.

The zero forcing number was introduced in $[1$ as an upper bound for maximum nullity for simple graphs, and has been extended to various other types of graphs. In fact, the definition is identical with the exception of the color-change rule, which varies with the type of graph. Since our matrices have all diagonal entries equal to zero, the color change rule for a loop graph with no loops 7 applies. This is the same as the color change rule used for the skew zero forcing number, denoted $\mathrm{Z}^{-}(G)$ [8]; the difference from the color change rule for standard zero forcing is that a vertex need not be colored to force. It is not surprising that the skew color change rule applies to symmetric matrices with zero diagonal, because zero forcing considers only the nonzero pattern of entries rather than the values of entries. In particular, we have $\mathrm{M}_{0}(G) \leq \mathrm{Z}^{-}(G)$. 7 .

An algorithm for computing the maximum nullity of a loop tree was established in 2], and of course this applies to computing the maximum zero-diagonal nullity of a (simple) tree. It is shown in 7 that the maximum nullity of a loop tree (including one without loops) is equal to its zero forcing number, so for a (simple) tree, $\mathrm{M}_{0}(T)=$ $\mathrm{Z}^{-}(G)$. The study of skew-symmetric matrices leads to an even simpler method for computing the maximum zero-diagonal nullity of a tree: For any (simple) tree $T$, 
$\operatorname{mr}_{0}(T)=2 \operatorname{match}(T)$, and $\operatorname{match}(T)$ can be determined by starting with a vertex of degree 1 , matching it, removing both matched vertices from the graph, and continuing in this manner 8 .

A vertex $v$ of a connected graph $G$ is a cut-vertex if $G-v$ is disconnected. If $G$ has a cut-vertex, it is well known that the problem of computing the (standard) minimum rank of $G$ can be reduced to computing minimum ranks of certain subgraphs. The cut-vertex reduction formula was extended to loop graphs in [10, and the version of the formula for graphs whose cut-vertex has no loop can be applied to reduce the problem of determining $\operatorname{mr}_{0}(G)$ for a graph $G$ with a cut-vertex. The reader is referred to [10] for the details.

2. Low minimum zero-diagonal rank. In this section, we characterize connected graphs whose minimum zero-diagonal rank is at most 3 .

2.1. Minimum zero-diagonal rank at most two. The next corollary follows immediately from Observation [1.1.2.

Corollary 2.1. Let $G$ be a connected graph. Then $\operatorname{mr}_{0}(G)=0$ if and only if $G$ is a single vertex.

The rank 1 case can be quickly handled as well, and the result is very different from standard minimum rank, where $\operatorname{mr}\left(K_{n}\right)=1$ for $n \geq 2$.

Proposition 2.2. There is no graph $G$ with $\operatorname{mr}_{0}(G)=1$.

Proof. Suppose that $A \in \mathcal{S}_{0}(G)$ and $\operatorname{rank} A \geq 1$. Then $A$ must have at least one nonzero entry, call it $a_{i j}$. Note that $A \in \mathcal{S}_{0}(G)$ implies that $i$ is distinct from $j$, $a_{j i}=a_{i j}$, and $a_{i i}=a_{j j}=0$. Thus, $\operatorname{rank} A \geq 2$.

TheOREM 2.3. Let $G$ be a connected graph with $|G| \geq 2$. Then the following are equivalent.

1. $\operatorname{mr}_{0}(G)=2$.

2. $G=K_{m, n}$.

3. $G$ does not contain an induced $C_{3}$ or $P_{4}$.

Proof. (2) $\Rightarrow$ (1): The adjacency matrix of $K_{m, n}$ has rank 2 , so $\operatorname{mr}_{0}\left(K_{m, n}\right) \leq 2$. Corollary 2.1 and Proposition 2.2 show that the minimum zero-diagonal rank of $K_{m, n}$ must be at least 2 . So $\operatorname{mr}_{0}\left(K_{m, n}\right)=2$.

(11) $\Rightarrow$ (3): Let $\operatorname{mr}_{0}(G)=2$. Then $G$ cannot contain $C_{3}$ or $P_{4}$ as an induced subgraph because $\operatorname{mr}_{0}\left(C_{3}\right)=3$ and $\mathrm{mr}_{0}\left(P_{4}\right)=4$ by Propositions 1.7 and 1.6 .

$(3) \Rightarrow(2)$ : Assume $G$ is a connected graph that does not contain $C_{3}$ or $P_{4}$ as an 
induced subgraph. Since $C_{k}$ has $P_{k-1}$ as an induced subgraph, $G$ cannot contain any induced $C_{k}$ for $k \geq 5$. Thus, $G$ has no odd cycles, and so $G$ is bipartite. Since $G$ is connected there is a path from any vertex to any other vertex. If $u$ and $v$ are in different partite sets, then the shortest path between them must have an even number of vertices, so if $u$ and $v$ were not adjacent then $G$ would have an induced $P_{4}$. Thus, $u$ and $v$ must be adjacent and $G$ is a complete bipartite graph.

2.2. Minimum zero-diagonal rank equal to three. In order to classify all graphs with $\operatorname{mr}_{0}(G)=3$, we first consider the family of complete graphs $K_{n}$. Since $K_{2}=P_{2}$ and $K_{3}=C_{3}$, we already have that $\operatorname{mr}_{0}\left(K_{2}\right)=2$ and $\operatorname{mr}_{0}\left(K_{3}\right)=3$. We define a family of Toeplitz matrices $T_{n} \in \mathcal{S}_{0}\left(K_{n}\right)$ by defining $\left(T_{n}\right)_{i, j}=(i-j)^{2}$. That is,

$$
T_{n}=\left[\begin{array}{ccccc}
0 & 1 & 4 & \cdots & (n-1)^{2} \\
1 & 0 & 1 & \cdots & (n-2)^{2} \\
4 & 1 & 0 & \cdots & (n-3)^{2} \\
\vdots & \vdots & \vdots & \ddots & \vdots \\
(n-1)^{2} & (n-2)^{2} & (n-3)^{2} & \cdots & 0
\end{array}\right]
$$

Theorem 2.4. For $T_{n}(n \geq 3)$ defined in (2.1), $\operatorname{rank} T_{n}=3$ and $\mathrm{mr}_{0}\left(K_{n}\right)=3$.

Proof. Since $K_{n}$ is not bipartite for $n \geq 3, \operatorname{mr}_{0}\left(K_{n}\right) \geq 3$. We show $\operatorname{rank} T_{n}=3$, thus establishing $\operatorname{mr}_{0}\left(K_{n}\right)=3$.

Let $r_{i}$ be the $i$ th row of $T_{n}$; then we claim that for all $4 \leq i \leq n$, the $i$ th row of $T_{n}$ is the following linear combination of the first three rows:

$$
r_{i}=\frac{1}{2}(i-2)(i-3) r_{1}-(i-1)(i-3) r_{2}+\frac{1}{2}(i-1)(i-2) r_{3} .
$$

This can be verified by checking that the $j$ th entries are equal, i.e.,

$(i-j)^{2}=\frac{1}{2}(i-2)(i-3)(1-j)^{2}-(i-1)(i-3)(2-j)^{2}+\frac{1}{2}(i-1)(i-2)(3-j)^{2}$.

We can now classify all graphs $G$ with minimum zero-diagonal rank $\operatorname{mr}_{0}(G)=3$.

TheOREm 2.5. For a connected graph $G, \mathrm{mr}_{0}(G)=3$ if and only if $G=$ $K_{n_{1}, n_{2}, \ldots, n_{r}}$ for some $r \geq 3$.

Proof. Assume $\mathrm{mr}_{0}(G)=3$. Then $G$ does not contain either $P_{4}$ or a paw (see Figure 1.1) as an induced subgraph, because each has a unique spanning generalized cycle and hence a minimum zero-diagonal rank of 4 by Remark 1.3. From Theorem 2.1 in $8, G$ does not contain $P_{4}$ or the paw as an induced subgraph if and only if 
$G=K_{n_{1}, n_{2}, \ldots, n_{r}}$ for some $r \geq 2$. Furthermore, $r \geq 3$, because $\operatorname{mr}_{0}(G)=3$ and we have already established that $\operatorname{mr}_{0}\left(K_{n_{1}, n_{2}}\right)=2$. Note that $K_{n}$ may also be thought of as the complete $n$-partite graph with one vertex in each partite set.

Conversely, let $G=K_{n_{1}, n_{2}, \ldots, n_{r}}, r \geq 3$. It remains to show that $\operatorname{mr}_{0}\left(K_{n_{1}, n_{2}, \ldots, n_{r}}\right)$ $=3$. We do this by exhibiting a matrix for $K_{n_{1}, n_{2}, \ldots, n_{r}}$ that achieves this minimum rank. By Theorem 2.4 $\operatorname{rank} T_{r}=3$. Let $T_{r}=\left[t_{i j}\right]$ and let $J_{k, \ell}$ denote the $k \times \ell$ matrix all of whose entries are 1 . We form the block matrix

$$
B=\left[\begin{array}{cccc}
0 & t_{12} J_{n_{1}, n_{2}} & \cdots & t_{1 r} J_{n_{1}, n_{r}} \\
t_{12} J_{n_{2}, n_{1}} & 0 & \cdots & t_{2 r} J_{n_{2}, n_{r}} \\
\vdots & \vdots & \ddots & \vdots \\
t_{1 r} J_{n_{r}, n_{1}} & t_{2 r} J_{n_{r}, n_{2}} & \cdots & 0
\end{array}\right]
$$

It is straightforward to see that $B \in \mathcal{S}_{0}\left(K_{n_{1}, n_{2}, \ldots, n_{r}}\right)$ and $\operatorname{rank} B=\operatorname{rank} T_{r}=3$.

3. High minimum zero-diagonal rank. In this section, we give a graphtheoretic characterization of graphs $G$ with $\operatorname{mr}_{0}(G)=|G|$ and then provide an algorithm that determines whether a graph satisfies that characterization, namely having a unique spanning generalized cycle (equivalently, a unique perfect [1,2]-factor).

\subsection{Graphs having minimum zero-diagonal rank equal to the order of the graph.}

THEOREM 3.1. Let $G$ be a bipartite graph. The following are equivalent:

1. G has a unique perfect matching.

2. $G$ has a unique spanning generalized cycle.

3. $\operatorname{mr}_{0}(G)=|G|$.

Proof. (1) $\Leftrightarrow$ (2): By definition a perfect matching is a spanning generalized cycle. Note that since $G$ is bipartite, $G$ has no odd cycle. If a spanning generalized cycle $\mathcal{C}$ of $G$ contains a cycle $C$, then $C$ is necessarily an even cycle. Since an even cycle has two perfect matchings, $\mathcal{C}$ would produce at least two perfect matchings of $G$.

$(2) \Leftrightarrow(3):(\Rightarrow)$ follows from Remark1.3 (for all graphs, not just bipartite graphs). For $(\Leftarrow)$ we prove the contrapositive. If $G$ has no spanning generalized cycle, then by Remark 1.4 $\operatorname{mr}_{0}(G)<|G|$. So suppose $G$ has at least two spanning generalized cycles. Then it was shown in the proof that (1) $\Leftrightarrow(2)$ that $G$ has at least two perfect matchings. Then by $[8$, Theorem 2.6], there exists a skew-symmetric matrix $B$ with the nonzero pattern described by $G$ and $\operatorname{rank} B<|G|$. Let the partite sets of $G$ be denoted by $U$ and $W$. Define a diagonal matrix $D=\left[d_{i j}\right]$ such that $d_{u u}=1$ 
for $u \in U$ and $d_{w w}=-1$ for $w \in W$. Then $D B$ is symmetric, $\mathcal{G}(D B)=G$ and $\operatorname{rank}(D B)=\operatorname{rank} B<|G|$, so $\mathrm{mr}_{0}(G)<|G|$. $\mathrm{Q}$

An odd cycle shows that (11) and (2) in Theorem 3.1 are not equivalent without the assumption that the graph is bipartite. We prove that the equivalence of (21) and (3) in Theorem 3.1 is true in general (see Theorem 3.9 below). We prove (3) implies (2) by contradiction. Suppose to the contrary that there is a graph $G$ satisfying $\operatorname{mr}_{0}(G)=|G|$ that does not have a unique spanning generalized cycle. Let $H_{*}=\left(V_{*}, E_{*}\right)$ be a minimum counterexample in the sense that every graph $G$ on fewer vertices than $\left|H_{*}\right|$ having $\operatorname{mr}_{0}(G)=|G|$ necessarily has a unique spanning generalized cycle, and every graph on $\left|H_{*}\right|$ vertices with fewer edges than $H_{*}$ fulfills this condition also. Denote the order of $H_{*}$ by $n_{*}$. We now investigate the properties of $H_{*}$. Observe that $H_{*}$ has at least two spanning generalized cycles, since at least one spanning generalized cycle is guaranteed by Remark 1.4

Observation 3.2. By the minimality of $H_{*}$, every edge of $H_{*}$ is included in some spanning generalized cycle of $H_{*}$.

Lemma 3.3. Let $C$ be a connected component (that is, a cycle or edge) in a spanning generalized cycle of $H_{*}$. Then there exists a spanning generalized cycle of $H_{*}$ that does not contain $C$.

Proof. Suppose every spanning generalized cycle of $H_{*}$ contains $C$. Without loss of generality, let the permutation associated with component $C$ be the cycle $(1 \cdots k)(k \geq 2)$. Then every spanning generalized cycle $\mathcal{C}$ of $H_{*}$ is of the form $C \dot{\cup} \mathcal{C}^{\prime}$ where $\mathcal{C}^{\prime}$ is a generalized cycle of order $\left(n_{*}-k\right)$ in $H_{*}\left[\left\{k+1, \ldots, n_{*}\right\}\right]$, so $H_{*}\left[\left\{k+1, \ldots, n_{*}\right\}\right]$ has more than one spanning generalized cycle. We will show that $\operatorname{mr}_{0}\left(H_{*}\left[\left\{k+1, \ldots, n_{*}\right\}\right]\right)=n_{*}-k$, which is the order of $H_{*}\left[\left\{k+1, \ldots, n_{*}\right\}\right]$, contradicting the minimality of $H_{*}$. Let $A \in \mathcal{S}_{0}\left(H_{*}\right)$. Then by (1.1),

$$
0 \neq \operatorname{det} A= \begin{cases}-a_{12} a_{21} \operatorname{det} A\left[\left\{k+1, \ldots, n_{*}\right\}\right] & \text { if } k=2, \\ (-1)^{k+1} 2 a_{12} \cdots a_{k-1, k} a_{k, 1} \operatorname{det} A\left[\left\{k+1, \ldots, n_{*}\right\}\right] & \text { if } k \geq 3,\end{cases}
$$

so $0 \neq \operatorname{det} A\left[\left\{k+1, \ldots, n_{*}\right\}\right]$. Since any element of $\mathcal{S}_{0}\left(H_{*}\left[\left\{k+1, \ldots, n_{*}\right\}\right]\right)$ can be realized as a principal submatrix of a matrix in $\mathcal{S}_{0}\left(H_{*}\right), \operatorname{mr}_{0}\left(H_{*}\left[\left\{k+1, \ldots, n_{*}\right\}\right]\right)=$ $n_{*}-k$, as desired.

We now include two technical lemmas that will help us prove Lemma 3.6. The first can be established by techniques in [8, Proposition 5.4] and the second can be established by application of the quadratic formula.

Lemma 3.4. If $p\left(x_{1}, \ldots, x_{q}\right)$ is a nonzero homogeneous polynomial over $\mathbb{R}$, then there exist nonzero real $c_{1}, \ldots, c_{q}$ such that $p\left(c_{1}, c_{2}, \ldots, c_{q}\right) \neq 0$.

Lemma 3.5. Suppose $m \geq 1$ is an integer and $p(z), q(z)$, and $s(z)$ are nonzero 
real polynomials satisfying $\operatorname{deg} p(z)=m, \operatorname{deg} q(z) \leq m$, and $\operatorname{deg} s(z) \leq m-1$. Then for $\alpha \in \mathbb{R}$ large enough, the quadratic equation $s(\alpha) x^{2}+p(\alpha) x+q(\alpha)=0$ has a nonzero real solution.

LEMma 3.6. A spanning generalized cycle of $H_{*}$ cannot contain an odd cycle.

Proof. We suppose $H_{*}$ contains a spanning generalized cycle $\mathcal{C}$ that contains an odd cycle $C$ and obtain a contradiction by constructing a matrix $B \in \mathcal{S}_{0}\left(H_{*}\right)$ with $\operatorname{det} B=0$, which implies $\operatorname{rank} B<n_{*}$ and so $\operatorname{mr}_{0}\left(H_{*}\right)<n_{*}$.

By Lemma 3.3, there exists a spanning generalized cycle $\mathcal{C}^{\prime}$ that does not contain $C$. So there is some edge $\{z, w\} \in E(C) \backslash E\left(\mathcal{C}^{\prime}\right)$. Let $t=\left|E_{*}\right|$ and $Y=\left[y_{u v}\right]$ be a symmetric matrix of indeterminates $x_{1}, x_{2}, \ldots, x_{t}$ with zero diagonal such that $\mathcal{G}(Y)=H_{*}\left(\right.$ so $\{u, v\} \in E_{*}$ implies $y_{u v}=y_{v u}=x_{i}$ for some $\left.x_{i}\right)$; without loss of generality, $y_{z w}=y_{w z}=x_{1}$ and the entries corresponding to the other edges of $\mathcal{C}$ are $x_{2}, \ldots, x_{\ell}$. Then the determinant of $Y$ is a homogeneous polynomial of degree $n_{*}$ in $x_{1}, x_{2}, \ldots, x_{t}$, and we can express $\operatorname{det} Y$ as

$$
\operatorname{det} Y=s\left(x_{2}, \ldots, x_{t}\right) x_{1}^{2}+p\left(x_{2}, \ldots, x_{t}\right) x_{1}+q\left(x_{2}, \ldots, x_{t}\right) .
$$

Since $\{z, w\} \notin E\left(\mathcal{C}^{\prime}\right), q\left(x_{2}, \ldots, x_{t}\right)$ is not identically zero. Furthermore, $p\left(x_{2}, \ldots, x_{t}\right)$ can be expressed as $h\left(x_{2}, \ldots, x_{\ell}\right)+g\left(x_{2}, \ldots, x_{t}\right)$, where every monomial in $g\left(x_{2}, \ldots, x_{t}\right)$ contains at least one variable not in $\left\{x_{2}, \ldots, x_{\ell}\right\}$; since the edges of $\mathcal{C}$ are represented by $\left\{x_{1}, \ldots, x_{\ell}\right\}, h\left(x_{2}, \ldots, x_{\ell}\right)$ is not identically zero. Thus, $p\left(x_{2}, \ldots, x_{t}\right)$ is not identically zero. By Lemma 3.4, we can choose nonzero $c_{2}, \ldots, c_{t}$ so that $h\left(c_{2}, \ldots, c_{\ell}\right) p\left(c_{2}, \ldots, c_{t}\right) q\left(c_{2}, \ldots, c_{t}\right) \neq 0$.

Define $B(\alpha)=\left[b_{u v}\right]$ to be the matrix obtained from $Y$ by replacing $y_{u v}=x_{i}$ by $\alpha c_{i}$ for $i=2, \ldots, \ell$, and $y_{u v}=x_{i}$ by $c_{i}$ for $i=\ell+1, \ldots, t$. For any polynomial $f\left(x_{2}, \ldots, x_{t}\right)$, define $\tilde{f}(\alpha)=f\left(\alpha c_{2}, \ldots, \alpha c_{\ell}, c_{\ell+1}, \ldots, c_{t}\right)$. Then

$$
\operatorname{det} B(\alpha)=\tilde{s}(\alpha) x_{1}^{2}+\tilde{p}(\alpha) x_{1}+\tilde{q}(\alpha) .
$$

If $s\left(c_{2}, \ldots, c_{t}\right)=0$, then we can solve $p\left(c_{2}, \ldots, c_{t}\right) x_{1}+q\left(c_{2}, \ldots, c_{t}\right)=0$ to obtain a nonzero value of $x_{1}$ that makes $\operatorname{det} B(1)=0$. So suppose $s\left(c_{2}, \ldots, c_{t}\right) \neq 0$. Any monomial in $\operatorname{det} Y$ has degree $n_{*}$, so $\operatorname{deg} s\left(x_{2}, \ldots, x_{t}\right)=n_{*}-2$, and thus, $\operatorname{deg} \tilde{s}(\alpha) \leq$ $n_{*}-2$. Because $\tilde{p}(\alpha)=\alpha^{n_{*}-1} h\left(c_{2}, \ldots, c_{\ell}\right)+\tilde{g}(\alpha)$ and $\operatorname{deg} \tilde{g}(\alpha)<n_{*}-1, \operatorname{deg} \tilde{p}(\alpha)=$ $n_{*}-1$.

If $\operatorname{deg} \tilde{q}(\alpha)=n_{*}$, then $H_{*}$ would necessarily contain a spanning generalized cycle whose edges are a subset of $E(\mathcal{C})$. Because $C$ is an odd cycle, it is not possible for any spanning generalized cycle that omits $\{z, w\}$ to have all its edges contained in $E(\mathcal{C})$. Thus, $\operatorname{deg} \tilde{q}(\alpha) \leq n_{*}-1$. Then by Lemma 3.5. for $\alpha$ sufficiently large there exists a real $x_{1}$ making $\operatorname{det} B(\alpha)=0$. 
Lemma 3.7. Any edge e of $H_{*}$ occurs as part of an even cycle of length at least four in some spanning generalized cycle of $H_{*}$.

Proof. Let $e$ be an edge of $H_{*}$. Then $e$ must be part of some spanning generalized cycle $\mathcal{C}$ by Observation 3.2. By Lemma 3.6. all the components of $\mathcal{C}$ are even. Since any even cycle of $\mathcal{C}$ can be replaced by the edges in one of its perfect matchings, $e$ must be contained in some spanning generalized cycle $\mathcal{M}$ of $H_{*}$ such that each component of $\mathcal{M}$ is an edge. By Lemma 3.3. $e$ is not an isolated edge of $H_{*}$. Let $f$ be an edge adjacent to $e$; $f$ must similarly be contained in a spanning generalized cycle $\mathcal{M}^{\prime}$ of $H_{*}$ whose components are all edges. Note that the adjacency of $e$ and $f$ guarantee that $\mathcal{M}^{\prime}$ is distinct from $\mathcal{M}$. Clearly the subgraph $\mathcal{M} \cup \mathcal{M}^{\prime}$ is a spanning subgraph of $H_{*}$. Any vertex $v$ of $H_{*}$ has degree one in both $\mathcal{M}$ and $\mathcal{M}^{\prime}$, so the degree of $v$ in $\mathcal{M} \cup \mathcal{M}^{\prime}$ is one or two. Moreover, each vertex $v$ is incident with exactly one edge in $\mathcal{M}$ and one in $\mathcal{M}^{\prime}$. If these edges are the same, the component containing $v$ in $\mathcal{M} \cup \mathcal{M}^{\prime}$ is an edge. If these edges are distinct, they must be part of a cycle that alternates edges of $\mathcal{M}$ and $\mathcal{M}^{\prime}$ and so the component containing $v$ in $\mathcal{M} \cup \mathcal{M}^{\prime}$ is an even cycle. Thus, $\mathcal{M} \cup \mathcal{M}^{\prime}$ is a spanning generalized cycle with only even components, and since $e$ and $f$ both appear in this union, they must appear as part of a even cycle of length at least four.

LEMMA 3.8. Let $G$ be a graph that is a union of even cycles each of length at least 4. There is a matrix $M$ in $\mathcal{S}_{0}(G)$ with $\operatorname{rank} M<|G|$.

Proof. Let $C_{1}, \ldots, C_{t}$ be even cycles of length at least 4 whose union is $G$. We will construct a matrix $M \in \mathcal{S}_{0}(G)$ such that the row sums of $M$ are 0 . If we set $\mathbb{1}=[1,1, \ldots, 1]^{T}$, then $M \mathbb{1}=\mathbf{0}$, so $\operatorname{rank} M<|G|$. Let $C=\left(v_{1}, v_{2}, \ldots, v_{2 k}\right)$ be one of $C_{1}, \ldots, C_{t}$. Define $M_{C}$ to be the matrix with $i j$-entry equal to

$$
\left(M_{C}\right)_{i j}=\left\{\begin{aligned}
(-1)^{\ell}, & \text { if }\{i, j\}=\left\{v_{\ell}, v_{\ell+1}\right\} \text { for } \ell=1, \ldots, 2 k-1 \text { or } \\
& \{i, j\}=\left\{v_{\ell}, v_{1}\right\} \text { for } \ell=2 k ; \\
0, & \text { otherwise. }
\end{aligned}\right.
$$

Note that the entries in each row of $M_{C}$ are either all 0 or are all 0 except for one entry equal to 1 and one entry equal to -1 , and hence, the sum of the entries in each row is 0 . Choose as $M$ a linear combination of the $M_{C_{i}}, i=1, \ldots, t$, so that there is no cancellation of nonzero entries. Thus, $M \in \mathcal{S}_{0}(G)$ and $\operatorname{rank} M<|G|$.

TheOREM 3.9. For every graph $G, \operatorname{mr}_{0}(G)=|G|$ if and only if $G$ has a unique spanning generalized cycle.

Proof. A graph $G$ that has a unique spanning generalized cycle must have $\operatorname{mr}_{0}(G)=|G|$ by Remark 1.3 . Suppose to the contrary that there exists a graph $G$ satisfying $\operatorname{mr}_{0}(G)=|G|$ that does not have a unique spanning generalized cycle. Let $H_{*}$ be a minimum such counterexample. By Lemma 3.7. $H_{*}$ is a union of its even 
cycles of length at least 4 . Then by Lemma $3.8 \mathrm{mr}_{0}\left(H_{*}\right)<\left|H_{*}\right|$, contradicting the definition of $H_{*}$. $\square$

In the case of skew-symmetric matrices, it is shown in [8, Theorem 2.6] that a graph has skew minimum rank equal to the order of the graph if and only if the graph has a unique perfect matching. The proof is achieved by using the fact that for a skew-symmetric matrix $A$, $\operatorname{det} A=(\operatorname{pf} A)^{2}$, where $\operatorname{pf} A$ is the pfaffian of $A$; the pfaffian measures perfect matchings. This is not applicable to symmetric matrices.

\subsection{Determination of whether a graph has a unique spanning gener-} alized cycle.

Here, we show that a graph with minimum rank equal to its order, and thus with a unique spanning generalized cycle, must have a vertex of degree 1 or be a disjoint union of one or more odd cycles, leading to an algorithm that tests whether a graph has this property (and finds the unique spanning generalized cycle if it does).

ObSERvation 3.10. If $G$ has a spanning subgraph that has more than one spanning generalized cycle, then $G$ has more than one spanning generalized cycle.

REMARK 3.11. Let $G$ be a graph on $n$ vertices. Suppose that $\mathcal{C}$ is a generalized cycle of $G$ of order $k$, and that there is a (not necessarily induced) subgraph of $G$ consisting of all $n-k$ remaining vertices that is an even path $P$. Then $G$ has a spanning generalized cycle that includes $\mathcal{C}$, constructed by adding every other edge of $P$ to $\mathcal{C}$ starting with either end vertex.

REMARK 3.12. Let $G$ be a graph with a spanning generalized cycle $\mathcal{C}$. Suppose that there is a subset of the components of $\mathcal{C}$, say $\left\{C_{1}, C_{2}, \ldots, C_{k}\right\}$, such that the subgraph induced by $V\left(C_{1}\right) \cup V\left(C_{2}\right) \bigcup_{k} \cdots \cup V\left(C_{k}\right)$ has a generalized cycle $\mathcal{D}$ of order $\left|V\left(C_{1}\right)\right|+\cdots+\left|V\left(C_{k}\right)\right|$ with $\mathcal{D} \neq \bigcup_{i=1} C_{i}$. Then $\mathcal{C}$ is not unique, because we can construct the spanning generalized cycle $\mathcal{C}^{\prime} \neq \mathcal{C}$ by simply replacing the components $\left\{C_{1}, C_{2}, \ldots, C_{k}\right\}$ in $\mathcal{C}$ with $\mathcal{D}$.

LEMMA 3.13. The following graphs have more than one spanning generalized cycle.

1. An odd cycle with a (possibly subdivided) chord.

2. A Hamiltonian graph that is not itself equal to an odd cycle.

3. Two vertex-disjoint odd cycles joined by a path or two odd cycles sharing exactly one vertex.

Proof. For (11), let $G$ consist of an odd cycle $C$ with path $Q$ from vertex $u$ to vertex $v$, where both $u$ and $v$ are on $C$. Since $C$ is an odd cycle, one path $P$ along 
$C$ from $u$ to $v$ is odd, and one, $P^{\prime}$, is even. Define a spanning generalized cycle $\mathcal{C}_{1}$ to be the cycle $P \cup Q$ together with every other edge of the even path $P^{\prime}-\{u, v\}$. In the case $Q$ is odd, $P \cup Q$ is even, so replacing the cycle $P \cup Q$ in $\mathcal{C}_{1}$ with alternating edges creates a new spanning generalized cycle by Remark 3.12. In the case $Q$ is even, define a spanning generalized cycle $\mathcal{C}_{2}$ to be the cycle $C$ together with every other edge of the even path $Q-\{u, v\}$.

For (21), an odd order Hamiltonian graph that is not itself a cycle is covered by (11), and an even Hamiltonian graph has at least three spanning generalized cycles: the Hamilton cycle itself and two that correspond to perfect matchings of the Hamilton cycle.

For (3), let $G$ consist of the two odd cycles, $C$ and $C^{\prime}$, joined by a path $P$. Denote the end vertices of the path $P$ by $u \in C$ and $u^{\prime} \in C^{\prime}$ ( $u=u^{\prime}$ is permitted). Again we need to consider two cases, based on the parity of $P$. Suppose first that $P$ is even, and define $\mathcal{C}_{1}$ to be $C$ and $C^{\prime}$ together with every other edge of the even path $P-\left\{u, u^{\prime}\right\}$ (if $|P|=2$ there are no such edges). Define $\mathcal{C}_{2}$ by starting with $u$ and taking every other edge along the path $P$ from $u$ to $u^{\prime}$. The remaining subgraph consists of two even paths, $C-u$ and $C^{\prime}-u^{\prime}$, and Remark 3.11 completes the construction. Finally, suppose $P$ is odd. Construct $\mathcal{C}$ by taking $C$ and every other edge of the even path consisting of $P-u$ and $C^{\prime}$ with one of the cycle edges incident with $u^{\prime}$ removed (in the case $P$ is a single vertex, then that vertex and both its incident edges are removed from $C^{\prime}$ ). To construct additional spanning generalized cycles, we can use the same process starting with cycle $C^{\prime}$.

Lemma 3.14. Let $G$ be a connected graph that has a spanning generalized cycle $\mathcal{C}$ with all components of $\mathcal{C}$ having at least 3 vertices. If $\mathcal{C}$ is the unique spanning generalized cycle of $G$ then $G$ is an odd cycle.

Proof. Suppose $\mathcal{C}$ is unique. All components of $\mathcal{C}$ are odd cycles, and $G$ cannot contain a (possibly subdivided) chord of any cycle in $\mathcal{C}$, by Lemma 3.13 and Remark 3.12 Let $C$ be a cycle in $\mathcal{C}$. Since $G$ is connected, if there is any other cycle in $\mathcal{C}$ other than $C$, there must be an edge in $\mathrm{G}$ from $C$ to some other such cycle. But this violates uniqueness of $\mathcal{C}$ by Lemma 3.13 and Remark 3.12. Thus, $G=C$ is an odd cycle.

THEOREM 3.15. Let $G$ be a connected graph with a unique spanning generalized cycle $\mathcal{C}$. Then either $G$ has a vertex of degree one, or $G$ is an odd cycle.

Proof. Let $G_{1}$ be the subgraph of $G$ induced by those vertices that are in a component of order 2 (an edge) in $\mathcal{C}$. Let $G_{2}$ be the subgraph of $G$ induced by those vertices that are in a component of order 3 or more (a cycle) in $\mathcal{C}$. If $G_{1}$ is empty, we know by Lemma 3.14 that $G$ is an odd cycle. So assume that $G_{1}$ is non-empty. 
Note that the set of components of order 2 in $\mathcal{C}$ gives a perfect matching of $G_{1}$. Call this matching $M$. Let $P$ be a maximal $M$-alternating path in $G_{1}$ with end vertices $u$ and $v$. By the maximality of $P$, we know that one of the following must hold.

1. The vertex $u$ has degree 1 in $G$.

2. There is an edge from $u$ to a vertex in $G_{2}$.

3. There is a non- $P$-edge from $u$ back to a vertex on the path $P$.

One of these cases must hold for $v$ as well. If Case 1 holds for either $u$ or $v$, the result is established, so we assume that Case 1 does not hold for either $u$ or $v$, and obtain a contradiction.

If Case 2 holds for both $u$ and $v$, let $w_{u}$ (respectively, $w_{v}$ ) denote the neighbor of $u$ (respectively, $v$ ) in $G_{2}$. If $w_{u}$ and $w_{v}$ are on different cycles in $G_{2}$, then we have a subgraph $H$ consisting of these two odd cycles joined by the path $P$ and edges $\left\{u, w_{u}\right\}$ and $\left\{v, w_{v}\right\}$. If $w_{u}$ and $w_{v}$ are both on one cycle in $G_{2}$, then we have a subgraph $H$ consisting of this cycle and its subdivided chord that is the path $P$ and edges $\left\{u, w_{u}\right\}$ and $\left\{v, w_{v}\right\}$. In either case, the existence of $H$ contradicts uniqueness of $\mathcal{C}$ by Lemma 3.13 and Remark 3.12. Therefore, both $u$ and $v$ cannot satisfy Case 2.

Note that $|P| \geq 4$ (since at least one of $u$ and $v$ must satisfy Case 3). By Remark 3.12 $G[V(P)]$ cannot contain an even cycle $C$ with the property that $V(P)=V(C)$ or $G[V(P) \backslash V(C)]$ has a perfect matching; in particular, neither $u$ nor $v$ can be adjacent to a vertex $w$ such that an even cycle is formed that consists of part or all of $P$ and the edge $\{u, w\}$ (or $\{v, w\})$.

Thus, $u$ and $v$ cannot be adjacent, because $P$ and the edge $\{u, v\}$ would form such an even cycle. Suppose that $u$ satisfies Case 2 and $v$ satisfies Case 3. Then $v$ is adjacent to some vertex on $P$ that is not $u$ (and this does not form an even cycle), and $u$ is adjacent to a cycle in $G_{2}$. This forms a subgraph consisting of two odd cycles joined by a path, violating the uniqueness of $\mathcal{C}$ by Lemma 3.13 and Remark 3.12

This leaves the case that both $u$ and $v$ satisfy Case 3 . That is, $u$ is adjacent to a vertex $w_{u}$ and $v$ is adjacent to a vertex $w_{v}$, where both are on the path $P$ and the paths from $u$ to $w_{u}$ and $v$ to $w_{v}$ (along $P$ ) are both odd. Thus, it is impossible that $w_{u}=w_{v}$, since $P$ is an even path by construction. If $w_{u}$ is closer on $P$ to $u$ than is $w_{v}$, then we have two odd cycles joined by a path $\left(w_{u}\right.$ to $\left.w_{v}\right)$, which again violates the uniqueness of $\mathcal{C}$. The remaining possibility is that the order of the vertices along the path is $u, w_{v}, w_{u}, v$, and the paths along $P$ from $u$ to $w_{u}$ and from $v$ to $w_{v}$ are both odd. Then the subgraph consisting of $P$ and the additional edges $\left\{u, w_{u}\right\}$ and $\left\{v, w_{v}\right\}$ is an even cycle $C$ with a (possibly subdivided) chord, with the edges of $C$ being the edge $\left\{u, w_{u}\right\}$, the edges of $P$ from $w_{u}$ to $v$, the edge $\left\{v, w_{v}\right\}$, and the edges 
of $P$ from $w_{v}$ to $u$. Since $P$ has an even number of vertices, the (possibly subdivided) chord along $P$ from $w_{v}$ to $w_{u}$ is even. This violates the uniqueness of $\mathcal{C}$ because either $V(C)=V(P)$ or there is a perfect matching of the even path obtained from the subdivided chord by deleting $w_{v}$ and $w_{u}$. $\mathrm{\square}$

As a consequence of Theorem 3.15 we have the following algorithm to determine whether a graph has a unique spanning generalized cycle (i.e., perfect [1,2]-factor) and if so to produce it.

\section{AlGORITHM 3.16. Unique spanning generalized cycle} Input: The graph $G$.

Output: True/False variable UNIQUE, and if UNIQUE = True, then the unique spanning generalized cycle $\mathcal{C}$ of $G$.

1. $\mathcal{C}=\emptyset$.

2. While $G$ has a degree one vertex:

A. Choose a degree one vertex $u$ of $G$.

B. Set $v:=$ the unique neighbor of $u$.

C. Delete $u$ and $v$ from $G$.

D. $\mathcal{C}:=\mathcal{C} \cup G_{u, v}$, where $G_{u, v}$ is the edge $\{u, v\}$ and its endpoints.

3. If $G$ is a union of vertex-disjoint odd cycles:

Then $\mathcal{C}:=\mathcal{C} \cup G$.

4. If $G$ is a union of vertex-disjoint odd cycles or $G=\emptyset$ :

Then $U N I Q U E=$ True;

Else $U N I Q U E=$ False.

4. Maximum rank and ranks in between. When studying the ranks of the family of symmetric matrices (that have free diagonal) with off-diagonal pattern described by a graph, one studies only minimum rank, because it is well known and easy to see that the maximum rank is the order of the graph $G$, and every rank between the minimum and maximum ranks is realizable: An $n \times n$ matrix $B$ with $\operatorname{rank} B=n$ can be constructed by choosing $0<\varepsilon<\frac{1}{n}$ and defining $B=\left[b_{i j}\right]$ with $b_{i i}=1, b_{i j}=\varepsilon$ for $i \neq j$ and $\{i, j\} \in E(G)$, and $b_{i j}=0$ otherwise. We can go from any matrix $B \in \mathcal{S}(G)$ to any matrix $A \in \mathcal{S}(G)$ in steps that change the rank by at most one at each step as follows: For each $\{i, j\} \in E(G)$ with $j>i$, add the matrix $S_{i j}$, where $S_{i j}[\{i, j\}]=\left[\begin{array}{ll}a_{i j}-b_{i j} & a_{i j}-b_{i j} \\ a_{i j}-b_{i j} & a_{i j}-b_{i j}\end{array}\right]$ and all other entries are zero; each $S_{i j}$ represents one step and $\operatorname{rank} S_{i j} \leq 1$. Call the resulting matrix $M=\left[m_{i j}\right]$. Then for $i=1, \ldots, n$, add the diagonal matrix $D_{i}$, where the $i i$-entry of $D_{i}$ is $a_{i i}-m_{i i}$ and all other entries are zero; each $D_{i}$ represents one step and $\operatorname{rank} D_{i} \leq 1$. Thus, we must pass through every rank in the transition from a maximum rank matrix $B$ to a minimum rank matrix $A$. 
However, when the diagonal is restricted to being zero, we can no longer use the preceding techniques; as it turns out, these results no longer hold. For example, there is no full rank matrix whose graph is an odd path. Thus, it is of interest to study maximum rank, and also which ranks between the minimum and maximum can be realized. Given a graph $G$, we say that $G$ allows rank $r$ if there is a matrix $A \in \mathcal{S}_{0}(G)$ such that $\operatorname{rank} A=r$; in this case $A$ is said to realize rank $r$ for $G$. In Theorem 4.4, we show that there are graphs that do not allow some intermediate ranks.

4.1. Maximum rank. The maximum zero-diagonal rank of a graph $G$ is

$$
\operatorname{MR}_{0}(G)=\max \left\{\operatorname{rank} A: A \in \mathcal{S}_{0}(G)\right\}
$$

THEOREM 4.1. Let $G$ be a graph and let $m$ denote the maximum order of a generalized cycle of $G$. Then $\operatorname{MR}_{0}(G)=m$.

Proof. The argument in Remark 1.4 shows that if $G$ has no generalized cycle of order greater than $m$, then $\operatorname{MR}_{0}(G) \leq m$. We now show the reverse inequality. Select any generalized cycle $\mathcal{C}=(V(\mathcal{C}), E(\mathcal{C}))$ of order $m$ in $G$. Let $G^{\prime}=G[V(\mathcal{C})]$ and let $Y$ be a symmetric matrix of indeterminates $x_{1}, x_{2}, \ldots, x_{q}$ with zero diagonal such that $\mathcal{G}(Y)=G^{\prime}\left(\right.$ so $\{u, v\} \in E(\mathcal{C})$ implies $y_{u v}=y_{v u}=x_{k}$ for some $\left.x_{k}\right)$. Then the determinant of $Y$ can be expressed as a nonzero homogeneous polynomial $\operatorname{det} Y=p\left(x_{1}, x_{2}, \ldots, x_{q}\right)$ of degree $m$ over $\mathbb{R}$. Define $B=\left[b_{u v}\right]$ to be the matrix obtained from $Y$ by replacing $x_{i}$ by $c_{i}$ chosen as in Lemma 3.4, so $\operatorname{det} B \neq 0$. Define $A=\left[a_{u v}\right] \in \mathcal{S}_{0}(G)$ by

$$
a_{u v}= \begin{cases}b_{u v} & \text { if }\{u, v\} \in E\left(G^{\prime}\right) \\ 1 & \text { if }\{u, v\} \in E(G) \text { and }\{u, v\} \notin E\left(G^{\prime}\right) \\ 0 & \text { if }\{u, v\} \notin E(G) .\end{cases}
$$

Since $B$ is a principal submatrix of $A$ and $\operatorname{rank} B=m, \operatorname{rank} A \geq m$ and thus $\operatorname{MR}_{0}(G) \geq m$.

The next result is immediate from Theorem 4.1 and Observation 1.5]

Corollary 4.2. For every graph $G, 2 \operatorname{match}(G) \leq \operatorname{MR}_{0}(G)$.

Since the maximum order of a generalized cycle of a subgraph (not necessarily induced) is less than or equal to the maximum order of a generalized cycle of the graph, applying Theorem 4.1 gives the next corollary.

Corollary 4.3. If $H$ is a subgraph of $G$ then $\operatorname{MR}_{0}(H) \leq \operatorname{MR}_{0}(G)$.

4.2. Realizable ranks. The next theorem shows that it is not always possible to realize every rank between $\operatorname{mr}_{0}(G)$ and $\operatorname{MR}_{0}(G)$ by a symmetric zero diagonal 
matrix having graph $G$.

THEOREM 4.4. Let $G$ be a bipartite graph. Then $\operatorname{mr}_{0}(G)$ and $\mathrm{MR}_{0}(G)$ are even. Furthermore, there exists a matrix in $\mathcal{S}_{0}(G)$ of rank $r$ if and only if $r$ is an even integer with $\mathrm{mr}_{0}(G) \leq r \leq \mathrm{MR}_{0}(G)$.

Proof. Let the two partite sets have order $a$ and $b$. If we label the vertices of $\mathrm{G}$ such that the vertices $1,2, \ldots, a$ correspond to the vertices in the partite set of order $a$, then $\mathcal{S}_{0}(G)$ is the set of matrices of the block form

$$
\left[\begin{array}{cc}
0 & M \\
M^{T} & 0
\end{array}\right]
$$

where $M$ is an $a \times b$ matrix. Clearly, $\operatorname{rank} A=2 \operatorname{rank} M$, so the allowable ranks of $A$ are completely determined by the allowable ranks of $M$. But it is well known that every possible rank between the minimum rank and maximum rank of the matrices described by a (not necessarily symmetric) nonzero pattern can occur, by changing one entry at a time to go from a matrix realizing minimum rank to one realizing maximum rank.

Lemma 4.5. Suppose $A \in \mathbb{R}^{n \times n}$ has the property that every row of $A$ has a nonzero entry. Then there exists a real vector $\mathbf{x}$ such that every entry of $A \mathbf{x}$ is nonzero and $\mathbf{x}^{T} A \mathbf{x} \neq 0$.

Proof. Let $\mathbf{a}_{i}^{T}$ denote the $i$ th row of $A$, and let $\mathbf{x}=\left[x_{i}\right]$. The $i$ th entry of $A \mathbf{x}$ is $\mathbf{a}_{i}^{T} \mathbf{x}$. Since every row has a nonzero entry, $q_{i}\left(x_{1}, \ldots, x_{n}\right):=\mathbf{a}_{i}^{T} \mathbf{x}$ is not identically zero; observe that the polynomial $q_{i}\left(x_{1}, \ldots, x_{n}\right)$ is homogeneous. Similarly, $p\left(x_{1}, \ldots, x_{n}\right):=$ $\mathbf{x}^{T} A \mathbf{x}$ is homogeneous and not identically zero. Thus, we can apply Lemma 3.4 to $\left(p q_{1} \cdots q_{n}\right)\left(x_{1}, \ldots, x_{n}\right)$ obtain a solution $\mathbf{x}=\left[c_{1}, \ldots, c_{n}\right]^{T}$ such that for $i=1, \ldots, n$ the $i$ th coordinate of $A \mathbf{x}, \mathbf{a}_{i}^{T} \mathbf{x}=q_{i}\left(c_{1}, \ldots, c_{n}\right) \neq 0$, and $\mathbf{x}^{T} A \mathbf{x}=p\left(c_{1}, \ldots, c_{n}\right) \neq 0$.

THEOREM 4.6. Suppose $H$ is a connected graph of order $n$, and that the graph $G$ is constructed from $H$ by adding a single vertex adjacent to every vertex of $H$. If $H$ allows rank $k$, then $G$ allows rank $k+1$.

Proof. Given $A \in \mathcal{S}_{0}(H)$ with $\operatorname{rank} A=k$, then we can construct a rank $k+1$ matrix $\tilde{A}$ in $\mathcal{S}_{0}(G)$ as follows. Without loss of generality, let the new vertex be $n+1$. Since $H$ is connected, every row of $A$ has a nonzero entry. Thus, by Lemma 4.5 we can choose a real vector $\mathbf{x}$ such that every entry of $A \mathbf{x}$ is nonzero and $\mathbf{x}^{T} A \mathbf{x} \neq 0$. For $B=\left[\begin{array}{cc}A & A \mathbf{x} \\ \mathbf{x}^{T} A & \mathbf{x}^{T} A \mathbf{x}\end{array}\right], B \in \mathcal{S}(G)$ and $\operatorname{rank} B=\operatorname{rank} A=k$. Define $\tilde{A}=\left[\begin{array}{cc}A & A \mathbf{x} \\ \mathbf{x}^{T} A & 0\end{array}\right]$. Then $\tilde{A} \in \mathcal{S}_{0}(G)$. Since $\mathbf{x}^{T} A \mathbf{x} \neq 0, \operatorname{rank} \tilde{A}=\operatorname{rank} A+1=k+1$.

Corollary 4.7. The complete graph $K_{n}$ allows all ranks $r$ such that $3 \leq r \leq n$. 
Proof. By Theorem 2.4, we know that $\mathrm{mr}_{0}\left(K_{n}\right)=3$. Thus, the corollary holds for $n=3$. Assume it holds for $n=k$, and consider $K_{k+1}$. We know that $\operatorname{mr}_{0}\left(K_{k+1}\right)=3$ from Theorem 2.4. By Theorem 4.6 and the inductive hypothesis, $K_{k+1}$ allows ranks 4 through $k+1$, which completes the proof.

5. Conclusion. We have determined the minimum zero-diagonal rank for the following families of graphs: trees, cycles, complete graphs, complete multipartite graphs, and hypercubes. We have characterized graphs having minimum zero-diagonal rank at most 3 and those having minimum zero-diagonal rank equal to the order of the graph, including providing an algorithm to test for a unique spanning generalized cycle. We have characterized maximum zero-diagonal rank in terms of generalized cycles, and investigated ranks allowed between the maximum and minimum. This paper only begins the study of minimum zero-diagonal rank.

Acknowledgements. The authors would like to thank the anonymous referee for helpful suggestions. Much of this research was done at an American Institute of Mathematics workshop held at the Institute for Computational and Experimental Research in Mathematics with support from the National Science Foundation, and at Iowa State University. The authors thank AIM, ICERM, NSF, and ISU for their support.

\section{REFERENCES}

[1] AIM Minimum Rank - Special Graphs Work Group (F. Barioli, W. Barrett, S. Butler, S.M. Cioabă, D. Cvetković, S.M. Fallat, C. Godsil, W. Haemers, L. Hogben, R. Mikkelson, S. Narayan, O. Pryporova, I. Sciriha, W. So, D. Stevanović, H. van der Holst, K. Vander Meulen, and A. Wangsness). Zero forcing sets and the minimum rank of graphs. Linear Algebra and its Applications, 428:1628-1648, 2008.

[2] L.M. DeAlba, T.L. Hardy, I.R.Hentzel, L. Hogben, and A. Wangsness. Minimum rank and maximum eigenvalue multiplicity of symmetric tree sign patterns. Linear Algebra and its Applications, 418:389-415, 2006.

[3] S. Fallat and L. Hogben. The minimum rank of symmetric matrices described by a graph: A survey. Linear Algebra and its Applications, 426:558-582, 2007.

[4] S.M. Fallat and L. Hogben. Chapter 46: Minimum Rank, Maximum Nullity, and Zero Forcing Number of Graphs. In Handbook of Linear Algebra, second edition, L. Hogben (editor), CRC Press, Boca Raton, FL, 2014.

[5] F. Harary. The determinant of the adjacency matrix of a graph. SIAM Review, 4:202-210, 1962.

[6] A. Hoffmann and L. Volkmann. On unique $k$-factors and unique $[1, k]$-factors in graphs. Discrete Mathematics, 278:127-138, 2004.

[7] L. Hogben. Minimum rank problems. Linear Algebra and its Applications, 432:1961-1974, 2010.

[8] IMA-ISU research group on minimum rank (M. Allison, E. Bodine, L.M. DeAlba, J. Debnath, L. DeLoss, C. Garnett, J. Grout, L. Hogben, B. Im, H. Kim, R. Nair, O. Pryporova, K. Savage, B. Shader, and A. Wangsness Wehe). Minimum rank of skew-symmetric matrices described by a graph. Linear Algebra and its Applications, 432:2457-2472, 2010.

[9] R. Merris. Graph Theory. Wiley, New York, 2001. 
Electronic Journal of Linear Algebra ISSN 1081-3810

A publication of the International Linear Algebra Society

Volume 27, pp. 458-477, June 2014

[10] R.C. Mikkelson. Minimum Rank of Graphs that Allow Loops. PhD Thesis, Iowa State University, 2008. Available at http://www.math.iastate.edu/thesisarchive/ PhD/MikkelsonPhDF08.pdf. 